\title{
High JAK2 Protein Expression Predicts a Poor Prognosis in Patients with Resectable Pancreatic Ductal Adenocarcinoma
}

\author{
Yang Song, ${ }^{1}$ Mei-Yue Tang, ${ }^{1}$ Wei Chen, ${ }^{1}$ Zhe Wang, ${ }^{2}$ and Si-Liang Wang ${ }^{1}{ }^{1}$ \\ ${ }^{1}$ Department of Oncology, Shengjing Hospital of China Medical University, 36 Sanhao Road, Shenyang 110004, China \\ ${ }^{2}$ Department of Pathology, Shengjing Hospital of China Medical University, 36 Sanhao Road, Shenyang 110004, China
}

Correspondence should be addressed to Si-Liang Wang; doctorwangsl@hotmail.com

Received 4 December 2019; Revised 4 September 2020; Accepted 8 September 2020; Published 22 September 2020

Academic Editor: Michele Malaguarnera

Copyright (C) 2020 Yang Song et al. This is an open access article distributed under the Creative Commons Attribution License, which permits unrestricted use, distribution, and reproduction in any medium, provided the original work is properly cited.

Background. Pancreatic ductal adenocarcinoma (PDAC) is one of the most fatal malignancies worldwide. The JAK/STAT signaling pathway is involved in pancreatic cancer tumorigenesis. However, the prognostic value of JAK2 expression in resectable PDAC is unclear. Method. In this study, we performed a clinicopathological analysis of 62 resectable PDAC cases with a primary focus on survival. JAK2 expression was examined by immunohistochemistry. The relationship between JAK2 expression and clinicopathological features and prognosis was analyzed. Results. Survival curve analyses revealed that high levels of JAK2 expression predict a poor prognosis in resectable PDAC patients. Multivariate analysis confirmed that JAK2 expression can predict the prognosis of PDAC. Conclusions. Assessment of JAK2 protein expression may be a promising method to predict prognosis in patients with resectable PDAC.

\section{Introduction}

Pancreatic ductal adenocarcinoma (PDAC) ranks fourth as the cause of tumor-related death and has 1-year and 5-year survival rates of $25 \%$ and $<5 \%$, respectively [1]. Compared with other cancer types, in which there has been a steady improvement in survival, little progress has been made in pancreatic cancer. Because the pancreas is retroperitoneal, tumor-associated symptoms do not appear until the tumor progresses to an advanced stage or until the development of distant metastasis, when surgical treatment is not indicated [2]. FOLFIRINOX- (5-FU, leucovorin, irinotecan, and oxaliplatin) based chemotherapy with fractionated external beam radiotherapy is the standard treatment for unresectable pancreatic cancer patients [3]. The combination of gemcitabine plus albumin-bound paclitaxel is a preferred option for metastatic patients [4]. However, the prognosis of these patients remains poor. To date, immune checkpoint inhibitors have not shown significant positive results in the treatment of pancreatic cancer. The use of exosome-based immunotherapies for PDAC treatment was recently proposed [5]. However, the biological behavior of tumor cells determines the rate of early recurrence and metastasis and the chemo- and radioresistance of pancreatic cancer [6].

Various biomarkers, such as serum carbohydrate antigen 19-9 (CA19-9) levels, noncoding RNAs [7], programmed cell death 1 /programmed cell death ligand 1 [8], and circulating tumor DNA [9], can predict the prognosis of PDAC. However, identifying a prognostic marker involved in the progression of pancreatic cancer would be valuable [10]. The Janus kinase/signal transducer and activator of transcription (JAK/STAT) signaling pathway plays a role in pancreatic cancer $[11,12]$ and is responsible for tumor initiation and progression [13]. JAK/STAT pathway inhibition is a novel treatment strategy targeting the proliferation and survival of pancreatic cancer cells [14] and the catabolic response to malignancy [15]. A previous study [16] showed that the JAK1/JAK2 inhibitor ruxolitinib has potential clinical benefit. However, whether JAK2 is a prognostic marker for PDAC remains unknown. 
The aim of this study was to explore the prognostic value of JAK2 expression in patients with resectable pancreatic cancer. Immunohistochemistry was used to evaluate JAK2 expression in human pancreatic cancer tissues, and its relationship with the clinical outcome was estimated. The findings of this study may improve our knowledge of the clinical significance and prognostic value of JAK2 expression in pancreatic cancer patients.

\section{Materials and Methods}

2.1. Patient Characteristics. The medical records of patients with resectable pancreatic carcinoma treated between January 2010 and December 2016 at Shengjing Hospital of China Medical University were retrospectively reviewed. The inclusion criteria were as follows: histopathologically confirmed PDAC postsurgery, no treatments prior to surgery, the absence of other malignant tumors, and complete clinicopathological data. Sixty-two patients with PDAC were enrolled in the study.

2.2. Bioinformatics Analysis. A database (https://ualcan.path .uab.edu/index.html), which is based on The Cancer Genome Atlas (TCGA) database, was used to evaluate the expression pattern of JAK2 in a large number of PDAC tissues. "JAK2" and "pancreatic ductal adenocarcinoma" were used as the filter parameters.

2.3. Immunohistochemistry. All PDAC tissue specimens were processed in the Department of Pathology at Shengjing Hospital (Shenyang, China). Samples were formalin-fixed and paraffin-embedded and cut and mounted on slides. The JAK2 primary antibody (catalog no. BA3398, Boster Biological Technology, Wuhan, China) was diluted to $1: 100$. Slides were incubated with a primary antibody in a working solution of $100 \mu \mathrm{L}$. Two experienced pathologists who had no access to the clinical data analyzed the staining results, which were quantified using a staining index (values, 0-12) determined by multiplying the score of staining intensity by the score of the positive area. The intensity was scored as follows: 0 , negative; 1, weak; 2, moderate; and 3, strong. The frequency of positive cells was defined as follows: 0 , less than $5 \% ; 1,5 \%$ to $25 \% ; 2,26 \%$ to $50 \% ; 3,51 \%$ to $75 \%$; and 4 , greater than $75 \%$. JAK2 staining was classified as low expression (0 to 7 ) or high expression (8 to 12 ).

2.4. Statistical Analysis. Quantitative data were expressed as averages and ranges, and categorical findings were expressed as numbers and percentages. The $t$-test was adopted to compare the differences between the subgroups if the quantitative data was normally distributed; otherwise, the Mann-Whitney $U$ test was used. Overall survival (OS) was defined as the period from the day of the surgery to either the last followup visit or the date of death. The log-rank test and KaplanMeier method were used for survival analysis, and the Cox regression model was used to explore the prognostic factors. $P<0.05$ was considered statistically significant. SPSS software version 19.0 (SPSS Inc., Chicago, IL, USA) was used to analyze the data.

\section{Results}

3.1. Patient Information. The demographic and clinicopathological data of the 62 patients are described in Table 1 . None of the patients dropped out from the follow-up, and by the end of the study, 35 patients $(56.5 \%)$ died. The median age was 59 years (range, 38-78 years). There were 38 men $(61.3 \%)$ and 24 women $(38.7 \%)$. The median of the tumor diameter $\left(P_{25}-P_{75}\right)$ is $4.28 \mathrm{~cm}$. According to the eighth edition of the pancreatic cancer AJCC staging system, $4 \mathrm{~cm}$ is used as the threshold for T2/T3 staging. It has been shown that $4 \mathrm{~cm}$ is the threshold in other studies of pancreatic cancer prognosis $[17,18]$. Therefore, in our study, $4 \mathrm{~cm}$ was selected as the cut-off of the tumor diameter. Patients in both groups accounted for half. Fifty (80.6\%) patients had moderately or well-differentiated tumors, whereas $12(19.4 \%)$ patients had poorly differentiated tumors, and 27 (43.5\%) patients had lymph node metastasis. CA19-9 serum level was $>37 \mathrm{U} / \mathrm{mL}$ in $49(72.0 \%)$ patients. The median OS was 17.9 months.

3.2. JAK2 Expression in Resectable PDAC Tissue Samples. JAK2 expression was analyzed by bioinformatics analysis, and the results are shown in Figure 1. According to TCGA samples, JAK2 expression was significantly elevated in PDAC tissues $(P<0.05)$. JAK2 expression was examined by immunohistochemistry in tumor tissues from 62 patients with resectable PDAC for further verification. JAK2-positive staining was defined as the distribution of brown-yellow particles in PDAC cells, as shown in Figure 2. A JAK2 staining intensity of 0 or 1 was observed in 27 (43.5\%) patients, whereas a staining intensity of 2 or 3 was observed in 35 (56.5\%) patients. Eight of the $12(67 \%)$ patients with poor tumor differentiation and 27/50 (54\%) of patients with wellor moderately differentiated tumors had a staining intensity of 2 or 3 .

3.3. Analysis of Overall Survival. Table 2 shows the log-rank test results. JAK2 expression $(P=0.009)$, serum CA19-9 levels $(P=0.006)$, and histological tumor differentiation $(P=0.014)$ were related to OS. Other characteristics did not influence OS, such as sex, age, maximum tumor diameter, location, primary tumor category $(\mathrm{pT})$, and pathological regional lymph node $(\mathrm{pN})$ category $(P>0.05)$. High JAK2 expression and serum CA19-9 levels $\geq 37 \mathrm{U} / \mathrm{mL}$ were significantly related to OS according to the multivariate Cox model (Figure 3). High JAK2 expression and serum CA19-9 levels $\geq 37 \mathrm{U} / \mathrm{mL}$ were related to shorter OS independently (Table 3). These data suggested that, in addition to the conventional prognostic factor serum CA19-9 level, JAK2 expression may act as a valuable biomarker to predict the prognosis of patients with resectable PDAC.

\section{Discussion}

The JAK/STAT signaling pathway is involved in many physiological processes, such as differentiation, cell growth, immune function, and hematopoiesis [19]. The JAK/STAT pathway is persistently activated in many malignant solid tumors including lung cancer [20], breast cancer [21], colorectal cancer [22], head and neck cancer [23], pancreatic 
TABLE 1: Baseline characteristics of evaluable patients $(N=62)$.

\begin{tabular}{|c|c|c|c|c|}
\hline \multirow{2}{*}{ Characteristics } & \multirow{2}{*}{ No. of cases } & \multicolumn{2}{|c|}{ JAK expression } & \multirow{2}{*}{$P$ value } \\
\hline & & Low & High & \\
\hline \multicolumn{5}{|l|}{ Gender } \\
\hline Men & $38(61.3)$ & 22 & 16 & 0.773 \\
\hline Women & $24(38.7)$ & 13 & 11 & \\
\hline \multicolumn{5}{|l|}{ Age (years) } \\
\hline$\geq 60$ & $28(45.2)$ & 16 & 12 & 0.921 \\
\hline$<60$ & $34(54.8)$ & 19 & 15 & \\
\hline \multicolumn{5}{|l|}{ Location } \\
\hline Head & $30(48.4)$ & 19 & 11 & 0.289 \\
\hline Body/tail & $32(51.6)$ & 16 & 16 & \\
\hline \multicolumn{5}{|c|}{ Maximum tumor diameter $(\mathrm{cm})$} \\
\hline$<4$ & $31(50.0)$ & 17 & 14 & 0.797 \\
\hline$\geq 4$ & $31(50.0)$ & 18 & 13 & \\
\hline \multicolumn{5}{|l|}{ CA19-9 (U/mL) } \\
\hline$<37$ & $13(21.0)$ & 4 & 9 & 0.035 \\
\hline$\geq 37$ & $49(72.0)$ & 31 & 18 & \\
\hline \multicolumn{5}{|l|}{ Differentiation } \\
\hline Well/moderate & $50(80.6)$ & 27 & 23 & 0.426 \\
\hline Poor & $12(19.4)$ & 8 & 4 & \\
\hline \multicolumn{5}{|l|}{ pT category } \\
\hline $\mathrm{pT} 1+\mathrm{pT} 2$ & $34(54.8)$ & 17 & 17 & 0.258 \\
\hline pT3+pT4 & $28(45.2)$ & 18 & 10 & \\
\hline \multicolumn{5}{|l|}{$\mathrm{pN}$ category } \\
\hline pNo & $35(56.5)$ & 22 & 13 & 0.001 \\
\hline $\mathrm{pN} 1$ & $27(43.5)$ & 6 & 21 & \\
\hline \multicolumn{5}{|c|}{ Circumferential resection margin } \\
\hline R0 & $46(74.2)$ & 27 & 19 & 0.545 \\
\hline $\mathrm{R} 1$ & $16(25.8)$ & 8 & 8 & \\
\hline \multicolumn{5}{|l|}{ Neural invasion } \\
\hline Yes & $23(37.1)$ & 13 & 10 & 0.993 \\
\hline No & $39(62.9)$ & 22 & 17 & \\
\hline \multicolumn{5}{|l|}{ Vascular invasion } \\
\hline Yes & $16(25.8)$ & 11 & 5 & 0.249 \\
\hline No & $46(74.2)$ & 24 & 22 & \\
\hline
\end{tabular}

cancer [24], and some hematological diseases [25]. In pancreatic cancer, abnormal activation of STAT3 is crucial for invasion and metastasis [26]. JAK2 expression is correlated with pancreatic tumor cell metastasis and invasion [27]. Moreover, the JAK/STAT pathway is involved in pancreatic tumor cell immune escape [28]. JAK2/STAT3 pathway inhibitors can prolong the survival of mice by slowing tumor growth and stromal modification, in addition to altering immune cell infiltration [29]. In a pancreatic cancer mouse model, activation of JAK2/STAT3 signaling promotes stromal formation in tumors and can lead to gemcitabine resistance [30]. These data indicated that this signaling pathway may be related to both the prognosis and treatment of cancers. To the best of our knowledge, this is the first study providing evidence that high JAK2 protein expression levels are closely related to poor prognosis in resectable PDAC patients.

In a previous study, we showed that inhibition of JAK2 at the mRNA and protein levels can hamper cell growth and promote cell apoptosis in vivo and in vitro [31]. In a recent study, our team found that JAK2 inhibition increases the radiosensitivity of pancreatic cancer cells [32]. TCGA analysis showed that JAK2 mRNA expression was different between tumor samples and normal samples, but there was no significant difference in survival data. Considering the biological aggressive nature of $\mathrm{PDAC}$, choosing a prognostic marker is of great importance to help clinicians select therapies that will avoid unnecessary toxicity. We explored the correlation between JAK2 expression, clinicopathological characteristics, and PDAC patient survival in our hospital 


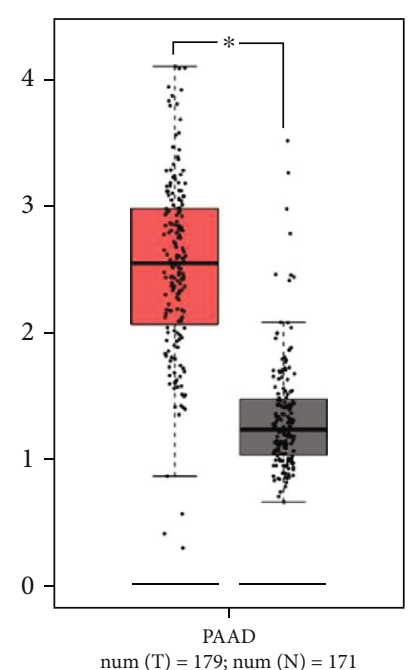

(a)

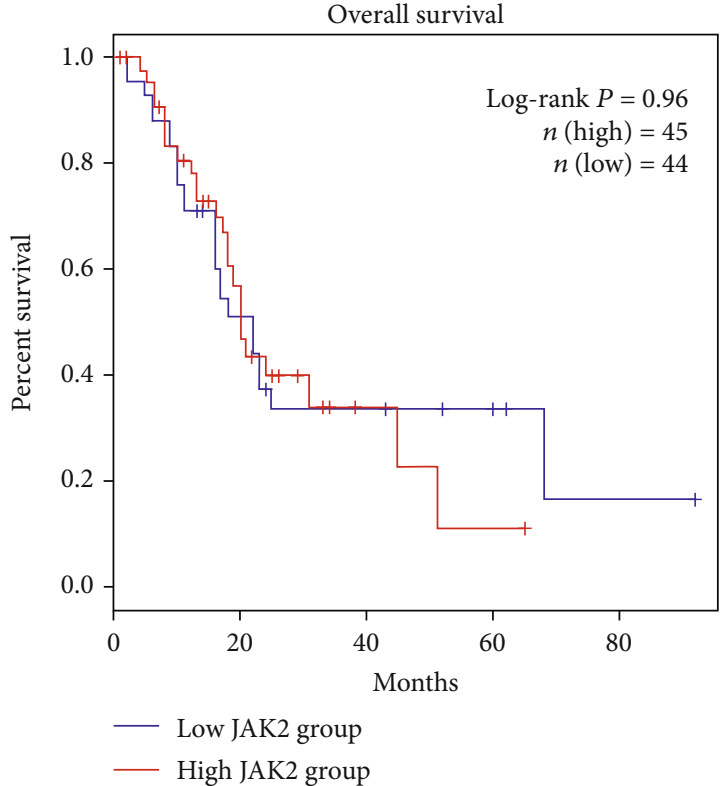

(b)

FIGURE 1: Using TCGA data to show (a) JAK2 mRNA expression and (b) the relationship between JAK2 mRNA and survival in PDAC tissues.

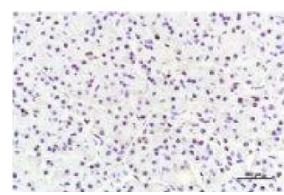

Negative (0)

(a)

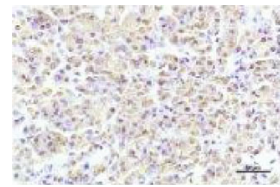

Moderate (2)

(c)

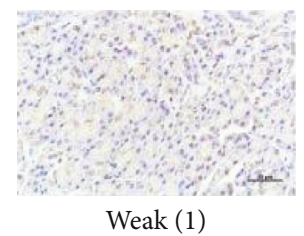

(b)

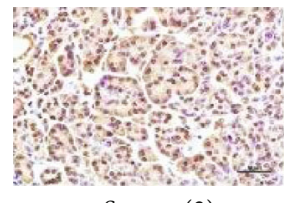

Strong (3)

(d)

FIGURE 2: Intensity of JAK2 expression in PDAC tissue by immunohistochemistry. Positive expression is shown by brown-yellow particles distributed in the cell membrane and cytoplasm (SP staining $\times 50)$. The cellular staining was classified using a scale of $0-3$ as follows: $(a)$ $0=$ negative, (b) $1=$ weakly positive, (c) $2=$ moderately positive, and (d) $3=$ strongly positive.

in this study. Various clinicopathological features including high serum CA19-9 levels and increased tumor size are significantly correlated with poor OS [33, 34]. Consistent with previous studies, the present results showed that tumor differentiation and serum CA19-9 levels were associated with OS. In addition, we showed that high JAK2 expression was associated with poor survival, indicating that JAK2 expression may act as a prognostic biomarker in resectable PDAC patients.

JAK2 is closely associated with not only pancreatic cancer initiation and progression but also radiosensitivity. The present findings suggested that the combination of CA19-9 level, histological tumor differentiation, and JAK expression may provide more accurate information for the prediction of prognosis in PDAC. However, this was a retrospective study, and the results need to be verified in a large cohort.

The results of bioinformatics analysis suggested that JAK2/STAT3 signaling was activated in pancreatic cancer tissues. Activated JAK2 phosphorylates STAT3, leading to its nuclear translocation and tumorigenesis [35]. A recent study showed that the natural compound B6 (3-deoxy$2 \beta$,16-dihydroxynagilactone E) inhibits the phosphorylation of STAT3 by inactivating and interacting with JAK2, thereby inhibiting growth and inducing apoptosis of breast cancer cells with overactivated STAT3; this provides a 
TABLE 2: Univariate analysis of overall survival (OS) and clinicopathological characteristics in 62 patients with resectable pancreatic ductal adenocarcinoma.

\begin{tabular}{|c|c|c|c|}
\hline Characteristics & Median OS (months) (95\% CI) & Log-rank $x^{2}$ & $P$ value \\
\hline Gender & & 0.05 & 0.824 \\
\hline Female & $20.5(18.9-22.0)$ & & \\
\hline Male & $22.4(19.4-25.4)$ & & \\
\hline Age (years) & & 0.244 & 0.621 \\
\hline$\geq 60$ & $24.6(18.9-30.3)$ & & \\
\hline$<60$ & $20.5(18.6-22.4)$ & & \\
\hline Location & & 0.055 & 0.815 \\
\hline Head & $20.5(17.2-23.8)$ & & \\
\hline Body/tail & $22.1(19.7-24.5)$ & & \\
\hline Maximum tumor diameter $(\mathrm{cm})$ & & 2.231 & 0.135 \\
\hline$<4$ & $24.6(17.6-31.6)$ & & \\
\hline$\geq 4$ & $20.8(17.0-24.5)$ & & \\
\hline CA19-9 (U/mL) level & & 7.561 & 0.006 \\
\hline$<37$ & $26.8(22.3-31.3)$ & & \\
\hline$\geq 37$ & $20.2(17.9-22.4)$ & & \\
\hline Differentiation & & 6.036 & 0.014 \\
\hline Well/moderate & $22.8(19.1-26.4)$ & & \\
\hline Poor & $13.8(6.97-20.6)$ & & \\
\hline pT category & & 0.008 & 0.928 \\
\hline $\mathrm{pT} 1+\mathrm{pT} 2$ & $21.3(18.4-24.2)$ & & \\
\hline $\mathrm{pT} 3+\mathrm{pT} 4$ & $22.1(18.0-26.1)$ & & \\
\hline pN category & & 0.973 & 0.324 \\
\hline pN0 & $20.5(17.8-23.2)$ & & \\
\hline $\mathrm{pN} 1$ & $25.8(17.7-33.9)$ & & \\
\hline JAK2 expression & & 6.761 & 0.009 \\
\hline Low & $24.6(17.8-31.4)$ & & \\
\hline High & $20.1(15.1-25.1)$ & & \\
\hline \multicolumn{4}{|l|}{ Circumferential resection margin } \\
\hline R0 & $20.8(18.3-23.3)$ & 0.062 & 0.803 \\
\hline $\mathrm{R} 1$ & $24.6(17.6-31.6)$ & & \\
\hline Neural invasion & & 1.166 & 0.280 \\
\hline Yes & $20.2(15.1-25.3)$ & & \\
\hline No & $22.8(17.5-28.1)$ & & \\
\hline Vascular invasion & & 3.725 & 0.054 \\
\hline Yes & $20.1(17.3-22.9)$ & & \\
\hline No & $22.8(17.3-28.3)$ & & \\
\hline
\end{tabular}

The $P$ value of the serum CA19-9 level, the histological differentiation, and the JAK2 expression is $\leq 0.05$, which means that they were significantly associated with OS.

novel promising strategy for the treatment of cancers with JAK2/STAT3 overactivation [36]. A Japanese study found that p-STAT3 expression was correlated with OS, and the activation of the JAK2/STAT3 pathway is critical for the survival of clear-cell ovarian cancers [37]. A different study suggested that combination of JAK2 inhibitors with immune checkpoint inhibition is effective for the treatment of non-small-cell lung cancer [38]. A dual inhibitor of STAT3 pathways induces death of tumor cells [39], indicating that suppression of the JAK2/STAT3 pathway may be a promising approach for the prevention and treatment of pancreatic cancer. As a key factor in the JAK/STAT pathway, JAK2 may be useful as a biomarker for predicting the prognosis of pancreatic cancer as well as a therapeutic target for patients with resectable PDAC.

In conclusion, JAK2 expression can serve as a novel, potent biomarker in resectable PDAC, providing independent prognostic information. The prognostic value of JAK2 expression can be improved through combination with clinical information, which supports patient risk 


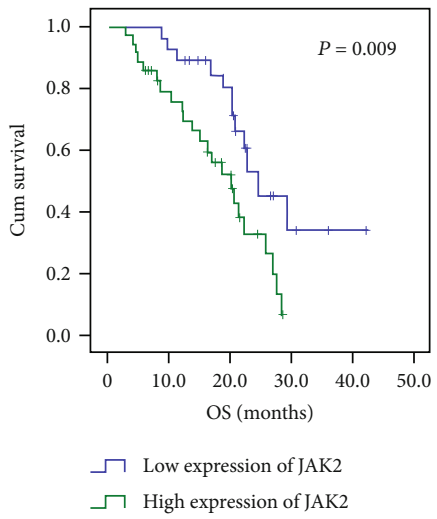

(a)

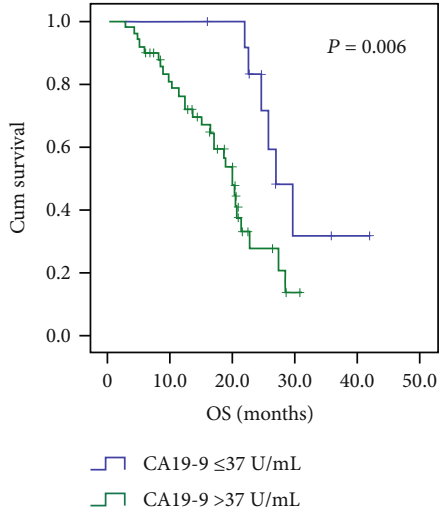

(b)

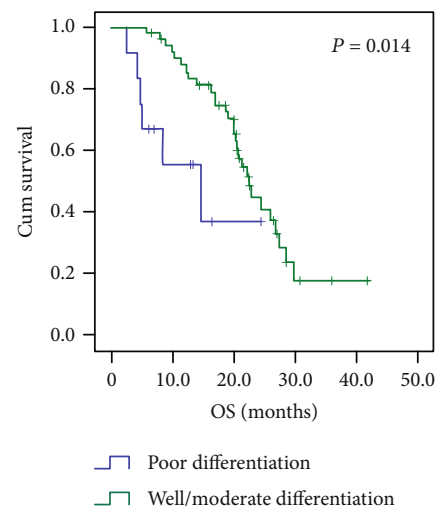

(c)

FIgURE 3: Overall survival (OS) in patients stratified by prognostic factors found to be independently associated with OS. (a) CA19-9 > 37 $\mathrm{U} / \mathrm{mL}$ predicts poor prognosis in patients with resectable pancreatic ductal adenocarcinoma. $P=0.006$. (b) Poor differentiation predicts poor prognosis in patients with resectable pancreatic ductal adenocarcinoma. $P=0.014$. (c) High expression of JAK2 predicts poor prognosis in patients with resectable pancreatic ductal adenocarcinoma. $P=0.009$. Significant differences in OS were revealed by the logrank test.

TABLE 3: Multivariate analysis of prognostic factors independently associated with overall survival (OS) in patients with resectable pancreatic ductal adenocarcinoma.

\begin{tabular}{|c|c|c|c|}
\hline Characteristics & Hazard ratio & $95 \% \mathrm{CI}$ & $P$ value \\
\hline CA19-9 (U/mL) & 0.298 & $0.116-0.769$ & 0.012 \\
\hline \multicolumn{4}{|l|}{$<37$} \\
\hline \multicolumn{4}{|l|}{$\geq 37$} \\
\hline Histological differentiation & 2.080 & $0.947-4.572$ & 0.068 \\
\hline \multicolumn{4}{|l|}{ Well/moderate } \\
\hline \multicolumn{4}{|l|}{ Poor } \\
\hline Expression of JAK2 & 0.476 & $0.230-0.988$ & 0.046 \\
\hline \multicolumn{4}{|l|}{ High } \\
\hline Low & & & \\
\hline
\end{tabular}

stratification and may be used for the design of different treatment strategies.

\section{Data Availability}

The Cancer Genome Atlas (TCGA) database data used to support the findings of this study are included within the article. The present survival data used to support the findings of this study are available from the corresponding author upon request.

\section{Ethical Approval}

The Ethics Committee of Shengjing Hospital of China Medical University (Shenyang, China) approved the study.

\section{Conflicts of Interest}

The authors declare that they have no conflict of interest.

\section{Acknowledgments}

This work was supported by funding from the Natural Science Foundation of Liaoning Province (201602863).

\section{References}

[1] R. L. Siegel, K. D. Miller, and A. Jemal, "Cancer statistics, 2019," CA: A Cancer Journal for Clinicians, vol. 69, no. 1, pp. 7-34, 2019.

[2] F. Eckel, T. Brunner, and S. Jelic, "Biliary cancer: ESMO Clinical Practice Guidelines for diagnosis, treatment and follow-up," Annals of Oncology, vol. 22, pp. vi40-vi44, 2011.

[3] M. A. Tempero, M. P. Malafa, S. W. Behrman et al., "Pancreatic adenocarcinoma, version 2.2014," Journal of the National Comprehensive Cancer Network, vol. 12, no. 8, pp. 10831093, 2014.

[4] M. Ducreux, T. Seufferlein, J. L. Van Laethem et al., "Systemic treatment of pancreatic cancer revisited," Seminars in Oncology, vol. 46, no. 1, pp. 28-38, 2019. 
[5] L. Huang, B. Ma, J. Ma, and F. Wang, "Fractalkine/CX3CR1 axis modulated the development of pancreatic ductal adenocarcinoma via JAK/STAT signaling pathway," Biochemical and Biophysical Research Communications, vol. 493, no. 4, pp. 1510-1517, 2017.

[6] T. Kamisawa, L. D. Wood, T. Itoi, and K. Takaori, "Pancreatic cancer," Lancet, vol. 388, no. 10039, pp. 73-85, 2016.

[7] S. C. Gupta and Y. N. Tripathi, "Potential of long non-coding RNAs in cancer patients: From biomarkers to therapeutic targets," International Journal of Cancer, vol. 140, no. 9, pp. 1955-1967, 2017.

[8] H. Wang, C. H. Lam, X. Li, D. L. West, and X. Yang, "Selection of PD1/PD-L1 X-Aptamers," Biochimie, vol. 145, pp. 125-130, 2018.

[9] D. Pietrasz, N. Pecuchet, F. Garlan et al., "Plasma circulating tumor DNA in pancreatic cancer patients is a prognostic marker," Clinical Cancer Research, vol. 23, no. 1, pp. 116123, 2017.

[10] Q. Dong, Y. Ma, Y. Zhang et al., "Cbl-b predicts postoperative survival in patients with resectable pancreatic ductal adenocarcinoma," Oncotarget, vol. 8, no. 34, pp. 57163-57173, 2017.

[11] A. Fukuda, S. C. Wang, J. P. Morris et al., "Stat3 and MMP7 Contribute to Pancreatic Ductal Adenocarcinoma Initiation and Progression," Cancer Cell, vol. 19, no. 4, pp. 441-455, 2011.

[12] D. von Ahrens, T. D. Bhagat, D. Nagrath, A. Maitra, and A. Verma, "The role of stromal cancer-associated fibroblasts in pancreatic cancer," Journal of Hematology \& Oncology, vol. 10, no. 1, p. 76, 2017.

[13] A. Quintas-Cardama and S. Verstovsek, "Molecular pathways: Jak/STAT pathway: mutations, inhibitors, and resistance," Clinical Cancer Research, vol. 19, no. 8, pp. 1933-1940, 2013.

[14] M. Lesina, M. U. Kurkowski, K. Ludes et al., "Stat 3/Socs 3 activation by IL- 6 transsignaling promotes progression of pancreatic intraepithelial neoplasia and development of pancreatic cancer," Cancer Cell, vol. 19, no. 4, pp. 456-469, 2011.

[15] A. Bonetto, T. Aydogdu, X. Jin et al., "JAK/STAT3 pathway inhibition blocks skeletal muscle wasting downstream of IL-6 and in experimental cancer cachexia," American Journal of Physiology. Endocrinology and Metabolism, vol. 303, no. 3, pp. E410-E421, 2012.

[16] H. I. Hurwitz, N. Uppal, S. A. Wagner et al., "Randomized, double-blind, phase II study of ruxolitinib or placebo in combination with capecitabine in patients with metastatic pancreatic cancer for whom therapy with gemcitabine has failed," Journal of Clinical Oncology, vol. 33, no. 34, pp. 4039-4047, 2015.

[17] P. J. Allen, D. Kuk, C. F. Castillo et al., "Multi-institutional Validation Study of the American Joint Commission on Cancer (8th Edition) Changes for T and N Staging in Patients With Pancreatic Adenocarcinoma," Annals of Surgery, vol. 265, no. 1, pp. 185-191, 2017.

[18] Z. Niu, M. Wang, L. Zhou, L. Yao, Q. Liao, and Y. Zhao, "Elevated GRP78 expression is associated with poor prognosis in patients with pancreatic cancer," Scientific Reports, vol. 5, no. 1, 2015.

[19] F. Perner, C. Perner, T. Ernst, and F. H. Heidel, "Roles of JAK2 in Aging, Inflammation, Hematopoiesis and Malignant Transformation," Cells, vol. 8, no. 8, p. 854, 2019.

[20] X. Yang, Z. Tang, P. Zhang, and L. Zhang, "Research advances of JAK/STAT signaling pathway in lung cancer," Zhongguo Fei Ai Za Zhi, vol. 22, no. 1, pp. 45-51, 2019.
[21] S. R. Hosford and T. W. Miller, "Clinical potential of novel therapeutic targets in breast cancer: CDK4/6, Src, JAK/STAT, PARP, HDAC, and PI3K/AKT/mTOR pathways," Pharmacogenomics and Personalized Medicine, vol. 7, pp. 203-215, 2014.

[22] T. Kusaba, T. Nakayama, K. Yamazumi et al., "Activation of STAT3 is a marker of poor prognosis in human colorectal cancer," Oncology Reports, vol. 15, no. 6, pp. 1445-1451, 2006.

[23] M. M. Choudhary, T. J. France, T. N. Teknos, and P. Kumar, "Interleukin-6 role in head and neck squamous cell carcinoma progression," World J Otorhinolaryngol Head Neck Surg., vol. 2, no. 2, pp. 90-97, 2016.

[24] A. Yakovenko, M. Cameron, and J. G. Trevino, "Molecular therapeutic strategies targeting pancreatic cancer induced cachexia," World J Gastrointest Surg., vol. 10, no. 9, pp. 95$106,2018$.

[25] T. A. Waldmann and J. Chen, "Disorders of the JAK/STAT pathway in T cell lymphoma pathogenesis: implications for immunotherapy," Annual Review of Immunology, vol. 35, no. 1, pp. 533-550, 2017.

[26] C. Huang, G. Yang, T. Jiang, K. Huang, J. Cao, and Z. Qiu, "Effects of IL-6 and AG490 on regulation of Stat 3 signaling pathway and invasion of human pancreatic cancer cells in vitro," Journal of Experimental \& Clinical Cancer Research, vol. 29, no. 1, p. 51, 2010.

[27] G. L. Razidlo, K. M. Burton, and M. A. McNiven, "Interleukin6 promotes pancreatic cancer cell migration by rapidly activating the small GTPase CDC42," Journal of Biological Chemistry, vol. 293, no. 28, pp. 11143-11153, 2018.

[28] T. Doi, T. Ishikawa, T. Okayama et al., "The JAK/STAT pathway is involved in the upregulation of PD-L1 expression in pancreatic cancer cell lines," Oncology Reports, vol. 37, no. 3, pp. 1545-1554, 2017.

[29] H. Yu, D. Pardoll, and R. Jove, "STATs in cancer inflammation and immunity: a leading role for STAT3," Nature Reviews. Cancer, vol. 9, no. 11, pp. 798-809, 2009.

[30] S. M. Wörmann, L. Song, J. Ai et al., "Loss of P53 Function Activates JAK2-STAT3 Signaling to Promote Pancreatic Tumor Growth, Stroma Modification, and Gemcitabine Resistance in Mice and Is Associated With Patient Survival," Gastroenterology, vol. 151, no. 1, pp. 180-193.e12, 2016.

[31] S. L. Wang, X. D. Chen, and M. Y. Tang, "MicroRNA-216a inhibits pancreatic cancer by directly targeting Janus kinase 2," Oncology Reports, vol. 32, no. 6, pp. 2824-2830, 2014.

[32] S. L. Wang, Y. J. Wang, S. M. Wu et al., "Identification of unique microRNAs associated with pancreatic cancer radioresistance using deep sequencing," Translational Cancer Research, vol. 7, no. 4, pp. 828-838, 2018.

[33] S. Boeck, C. Wittwer, V. Heinemann et al., "Cytokeratin 19fragments (CYFRA 21-1) as a novel serum biomarker for response and survival in patients with advanced pancreatic cancer," British Journal of Cancer, vol. 108, no. 8, pp. 16841694, 2013.

[34] J. Hlavsa, F. Cecka, P. Zaruba et al., "Tumor grade as significant prognostic factor in pancreatic cancer: validation of a novel TNMG staging system," Neoplasma, vol. 65, no. 4, pp. 637-643, 2018.

[35] R. Roskoski, "Janus kinase (JAK) inhibitors in the treatment of inflammatory and neoplastic diseases," Pharmacological Research, vol. 111, pp. 784-803, 2016.

[36] H. Shan, S. Yao, Y. Ye, and Q. Yu, “3-Deoxy-2 $\beta$,16-dihydroxynagilactone E, a natural compound from Podocarpus nagi, 
preferentially inhibits JAK2/STAT3 signaling by allosterically interacting with the regulatory domain of JAK2 and induces apoptosis of cancer cells," Acta Pharmacologica Sinica, vol. 40, no. 12, pp. 1578-1586, 2019.

[37] T. Yoshikawa, M. Miyamoto, T. Aoyama et al., "JAK2/STAT3 pathway as a therapeutic target in ovarian cancers," Oncology Letters, vol. 15, no. 4, pp. 5772-5780, 2018.

[38] S. P. Pitroda, M. E. Stack, G. F. Liu et al., "JAK2 inhibitor SAR302503 abrogates PD-L1 expression and targets therapyresistant non-small cell lung cancers," Molecular Cancer Therapeutics, vol. 17, no. 4, pp. 732-739, 2018.

[39] S. Kuang, C. Qi, J. Liu et al., "2-Methoxystypandrone inhibits signal transducer and activator of transcription 3 and nuclear factor- $\kappa \mathrm{B}$ signaling by inhibiting Janus kinase 2 and $\mathrm{I} \kappa \mathrm{B}$ kinase," Cancer Science, vol. 105, no. 4, pp. 473-480, 2014. 